\title{
Many-particle correlations and boundary conditions in the quantum kinetic theory
}

\author{
V.G.Morozov ${ }^{1}$, G.Röpke ${ }^{2}$ \\ 1 MIREA, Physics Department, \\ Vernadsky Prospect, 78, 117454 Moscow, Russia \\ 2 FB Physik, Universität Rostock, D-18051 Rostock, Germany
}

Received November 17, 1997

\begin{abstract}
The problem of many-particle correlations in different approaches to the quantum kinetic theory is treated on the basis of Zubarev's method of the nonequilibrium statistical operator. It is shown that long-lived correlations can be incorporated through boundary conditions for reduced manyparticle density matrices and the nonequilibrium real-time Green functions. Within the method of Green functions the boundary conditions are conveniently formulated in terms of the "mixed" Green functions defined on a directed contour with the real-time evolution governed by the Hamiltonian of the system and the "imaginary-time" evolution governed by the entropy operator. The perturbation expansion of the mixed Green function is obtained in terms of the interaction part of the Hamiltonian and the correlation part of the entropy operator.
\end{abstract}

Key words: nonequilibrium statistical operator, many-particle correlations, nonequilibrium Green functions, the entropy operator

PACS: $05.30 . C h, 05.20 . D d$

\section{Introduction}

At the present time there are two most-used approaches to nonequilibrium statistical mechanics of quantum systems, namely, the method based on the Liouvillevon Neumann equation for the statistical operator and the method of Green functions. In a sense, these two approaches correspond to the Schrödinger and Heisenberg pictures in quantum mechanics. It can be easily seen from obvious relations for the mean values of dynamical variables

$$
\langle\hat{A}\rangle^{t}=\operatorname{Tr}\{\hat{A} \varrho(t)\}=\operatorname{Tr}\left\{\hat{A}_{\mathrm{H}}(t) \varrho\left(t_{0}\right)\right\}
$$


where $t_{0}$ is some initial instant of time,

$$
\varrho(t)=U\left(t, t_{0}\right) \varrho\left(t_{0}\right) U\left(t_{0}, t\right)
$$

is the statistical operator in the Schrödinger picture, and

$$
\hat{A}_{\mathrm{H}}(t)=U\left(t_{0}, t\right) \hat{A} U\left(t, t_{0}\right)
$$

is the dynamical variable $\hat{A}$ in the Heisenberg picture. In the case when the Hamiltonian of the system $H$ does not depend explicitly on time, the evolution operator is given by

$$
U\left(t, t_{0}\right)=\mathrm{e}^{-\mathrm{i}\left(t-t_{0}\right) H / \hbar}
$$

Note that in the both pictures we are up against the problem of the initial statistical distribution $\varrho\left(t_{0}\right)$. Since there are no physical reasons to place a strong emphasis on the special time $t_{0}$, it is usual to assume that $t_{0} \rightarrow-\infty$, i.e. the evolution of the system starts in a distant past. Thus, the problem should be more properly referred to as the problem of boundary conditions for the statistical operator $\varrho(t)$.

It is widely believed that the problem of boundary conditions in nonequilibrium statistical mechanics is not of great importance, because a many-particle system "forgets" the details of its initial state and thus, as $t_{0} \rightarrow-\infty$, the choice of the limiting distribution $\varrho\left(t_{0}\right)$ is only a matter of convenience. However, the situation is not so simple and the purely formal limit $t_{0} \rightarrow-\infty$ has no justification, except "to take the whole history of the system into account". In practice the evolution of the system is usually described in terms of some quantities $Q_{m}(t)$ which vary slowly in time. Thus, in fact, we always deal with two quite different time scales. The first (shorter) scale is determined by some relaxation time $\tau_{r}$ which is the time for the establishment of a nonequilibrium (macroscopic) state described by the set $\left\{Q_{m}(t)\right\}$, and the second (larger) scale is determined by the characteristic time interval $\Delta t$ on which the quantities $Q_{m}(t)$ change. In other words, we have a hierarchy of time scales reflecting a hierarchy of relaxation times. This is nothing but the idea for the reduced description of many-particle systems, proposed and used by Bogoliubov for constructing kinetic equations on the basis of the Liouville equation [1]. In view of the above considerations, it is clear that the form of the initial distribution $\varrho\left(t_{0}\right)$ is irrelevant only for the description of rapidly damping correlations, i.e. the correlations existing on the shortest time scale $\tau_{r}$. As to longlived correlations associated with observables $Q_{m}(t)$, the meaning of the formal limit $t_{0} \rightarrow-\infty$ must be investigated more carefully. Specifically this has to do with the role of many-particle correlations arising from the evolution of locally conserved quantities.

In this paper, we want to discuss some general approaches to the problem of boundary conditions in the Schrödinger and the Heisenberg pictures of nonequilibrium statistical mechanics. For definiteness, we restrict our consideration to the quantum kinetic theory, where the difference between these two pictures has some interesting aspects. In particular, we shall see that, in the context of the kinetic theory, the problem of boundary conditions for the statistical operator is closely connected with the problem of many-particle correlations. 


\section{Boundary conditions in the Schrödinger picture of the quan- tum kinetic theory}

Recall that the main objective in nonequilibrium statistical mechanics is to derive transport equations for observables $f_{m}(t)=\left\langle P_{m}\right\rangle^{t}$ which are the mean values of some relevant dynamical variables $P_{m}$. Setting $\hat{A}=P_{m}$ in equations (1.1) and then differentiating these identities with respect to time, we obtain in the Schrödinger picture

$$
\frac{\partial}{\partial t}\left\langle P_{m}\right\rangle^{t}=\operatorname{Tr}\left\{P_{m} \frac{\partial \varrho(t)}{\partial t}\right\}
$$

The time derivative of the statistical operator can be eliminated by means of the Liouville-von Neumann equation

$$
\frac{\partial \varrho(t)}{\partial t}+\frac{1}{\mathrm{i} \hbar}[\varrho(t), H]=0 .
$$

Then, using the cyclic invariance of the trace, we arrive at the set of generalized transport equations

$$
\frac{\partial}{\partial t}\left\langle P_{m}\right\rangle^{t}=\operatorname{Tr}\left\{\dot{P}_{m} \varrho(t)\right\}
$$

where

$$
\dot{P}_{m}=\frac{1}{\mathrm{i} \hbar}\left[P_{m}, H\right]
$$

are the time derivatives of the relevant variables. They are also called the generalized fluxes.

The choice of observables and, consequently, of the relevant variables depends on the time scale used for the description of nonequilibrium states of the system (see, e.g., [2,3]). In the quantum kinetic theory, the most important observable is the single-particle density matrix

$$
f_{1}\left(l, l^{\prime} ; t\right)=\left\langle a_{l^{\prime}}^{\dagger} a_{l}\right\rangle^{t}
$$

where $a_{l}^{\dagger}$ and $a_{l}$ are (Bose or Fermi) creation and annihilation operators for a complete set of single-particle quantum states $|l\rangle$. It is clear that the role of relevant dynamical variables is played by the operators $P_{l l^{\prime}}=a_{l^{\prime}}^{\dagger} a_{l}$. Thus, in the case under consideration, equation (2.3) takes the form of a generalized kinetic equation

$$
\frac{\partial f_{1}\left(l, l^{\prime} ; t\right)}{\partial t}=\frac{1}{\mathrm{i} \hbar} \operatorname{Tr}\left\{\left[a_{l^{\prime}}^{\dagger} a_{l}, H\right] \varrho(t)\right\} .
$$

To calculate the averages on the right-hand side of this equation, we have to specify the Hamiltonian of the system and find the nonequilibrium statistical operator $\varrho(t)$. In what follows, we assume that the Hamiltonian has the form:

$$
H=\sum_{l l^{\prime}} h_{1}^{0}\left(l^{\prime}, l\right) a_{l^{\prime}}^{\dagger} a_{l}+\frac{1}{2} \sum_{l_{1} l_{2} l_{1}^{\prime} l_{2}^{\prime}} \Phi_{2}\left(l_{1}^{\prime} l_{2}^{\prime}, l_{1} l_{2}\right) a_{l_{2}^{\prime}}^{\dagger} a_{l_{1}^{\prime}}^{\dagger} a_{l_{1}}^{\dagger} a_{l_{2}},
$$


where $h_{1}^{0}\left(l, l^{\prime}\right)$ is a Hermitian single-particle energy matrix. The last term in equation (2.7) describes pairwise interactions between particles. The interaction amplitude possesses the usual symmetry properties

$$
\begin{gathered}
\Phi_{2}^{*}\left(l_{1}^{\prime} l_{2}^{\prime}, l_{1} l_{2}\right)=\Phi_{2}\left(l_{1} l_{2}, l_{1}^{\prime} l_{2}^{\prime}\right), \\
\Phi_{2}\left(l_{1}^{\prime} l_{2}^{\prime}, l_{1} l_{2}\right)=\mp \Phi_{2}\left(l_{1}^{\prime} l_{2}^{\prime}, l_{2} l_{1}\right)=\mp \Phi_{2}\left(l_{2}^{\prime} l_{1}^{\prime}, l_{1} l_{2}\right)
\end{gathered}
$$

with the upper sign for fermions and the lower sign for bosons.

Making use of the explicit form of the Hamiltonian and calculating the commutator on the right-hand side of equation (2.6), we find that the equation for the single-particle density matrix is not closed, since the interaction term gives rise to a two-particle density matrix. Then, setting $P_{m}=a_{l_{2}^{\prime}}^{\dagger} a_{l_{1}^{\prime}}^{\dagger} a_{l_{1}} a_{l_{2}}$ in equation (2.3), we obtain the evolution equation for the two-particle density matrix with the threeparticle density matrix on the right-hand side, etc. In other words, we have to consider the hierarchy of equations for reduced $n$-particle density matrices

$$
f_{n}\left(l_{1}^{\prime} \ldots l_{n}^{\prime}, l_{1} \ldots l_{n} ; t\right)=\left\langle a_{l_{n}^{\prime}}^{\dagger} \ldots a_{l_{1}^{\prime}}^{\dagger} a_{l_{1}} \cdots a_{l_{n}}\right\rangle^{t}, \quad n \geqslant 1,
$$

which is analogous to the well-known BBGKY hierarchy in the classical kinetic theory. Since all the equations in the quantum BBGKY hierarchy are differential equations with respect to time, one has to formulate some initial or boundary conditions for the reduced density matrices. The most elegant way is to formulate these conditions using a boundary condition for the nonequilibrium statistical operator $\varrho(t)$. In Zubarev's method [2,3], the boundary condition for $\varrho(t)$ is introduced by an infinitesimal source term breaking the time reversal symmetry of the Liouville-von Neumann equation. Formally, this means that we consider, instead of equation (2.2), the equation

$$
\frac{\partial \varrho(t)}{\partial t}+\frac{1}{\mathrm{i} \hbar}[\varrho(t), H]=-\varepsilon\left\{\varrho(t)-\varrho_{\mathrm{rel}}(t)\right\},
$$

where $\varepsilon \rightarrow+0$ after the thermodynamic limit in the averages calculated with $\varrho(t)$. The auxiliary relevant statistical operator $\varrho_{\text {rel }}(t)$ corresponds to the generalized Gibbs state described by some set of observables $\left\langle\hat{A}_{i}\right\rangle^{t}$. The explicit expression for $\varrho_{\text {rel }}(t)$ is found from the maximum of the information entropy for the given mean values $\left\langle\hat{A}_{i}\right\rangle^{t}[2,3]$ :

$$
\varrho_{\text {rel }}(t)=\exp \left(-\sum_{i} B_{i}(t) \hat{A}_{i}\right) / \operatorname{Tr} \exp \left(-\sum_{i} B_{i}(t) \hat{A}_{i}\right)
$$

where the Lagrange multipliers $B_{i}(t)$ are expressed in terms of $\left\langle\hat{A}_{j}\right\rangle^{t}$ from the self-consistency conditions

$$
\left\langle\hat{A}_{i}\right\rangle^{t}=\operatorname{Tr}\left\{\varrho_{\text {rel }}(t) \hat{A}_{i}\right\} .
$$

We recall that in the second-quantized form any dynamical variable can be written as a cluster decomposition:

$$
\hat{A}=\sum_{k \geqslant 1} \sum_{l_{1} \ldots l_{k} l_{1}^{\prime} \ldots l_{k}^{\prime}} A\left(l_{1}^{\prime} \ldots l_{k}^{\prime}, l_{1} \ldots l_{k}\right) a_{l_{k}^{\prime}}^{\dagger} \cdots a_{l_{1}^{\prime}}^{\dagger} a_{l_{1}} \cdots a_{l_{k}} .
$$


Thus, the most general form of the relevant statistical operator is $[4,5]$ :

$$
\varrho_{\text {rel }}(t)=\frac{1}{Z(t)} \exp \left(-\sum_{n \geqslant 1} s_{n}\left(l_{1}^{\prime} \ldots l_{n}^{\prime}, l_{1} \ldots l_{n} ; t\right) a_{l_{k}^{\prime}}^{\dagger} \cdots a_{l_{1}^{\prime}}^{\dagger} a_{l_{1}} \cdots a_{l_{n}}\right)
$$

(with additional summation over repeated arguments) ${ }^{1}$. The quantity $Z(t)$ is a normalization constant and the Lagrange multipliers $s_{n}$ are determined from the self-consistency conditions

$$
f_{n}\left(l_{1} \ldots l_{n}, l_{1}^{\prime} \ldots l_{n}^{\prime} ; t\right)=\operatorname{Tr}\left\{\varrho_{\text {rel }}(t) a_{l_{n}^{\prime}}^{\dagger} \cdots a_{l_{1}^{\prime}}^{\dagger} a_{l_{1}} \cdots a_{l_{n}}\right\}
$$

for the given $n$-particle density matrices. If the summation in equation (2.14) is over $1 \leqslant n \leqslant n_{\max }$, then the density matrices $f_{n}$ with $n \leqslant n_{\max }$ are considered as independent state parameters and the higher-order density matrices can be expressed in terms of them. Thus, the relevant statistical operator can describe a nonequilibrium system with many-particle correlations up to any order. Relations (2.15) play the role of nonequilibrium equations of state. Note that, in general, the explicit solution of these equations for thermodynamic parameters $s_{k}$ in terms of the reduced density matrices $f_{n}$ is a difficult problem, since infinite resummations of diagrams in the perturbation theory may be required [8]. In the special case $n_{\max }=1$, the single-particle density matrix is the only independent state parameter. The corresponding relevant distribution

$$
\varrho_{\text {rel }}(t)=\exp \left(-\sum_{l l^{\prime}} s_{1}\left(l^{\prime}, l ; t\right) a_{l^{\prime}}^{\dagger} a_{l}\right) / \operatorname{Tr} \exp \left(-\sum_{l l^{\prime}} s_{1}\left(l^{\prime}, l ; t\right) a_{l^{\prime}}^{\dagger} a_{l}\right)
$$

describes an ideal quantum gas. In this case, the parameters $s_{1}\left(l^{\prime}, l ; t\right)$ can be easily expressed in terms of the single-particle density matrix [3].

Now the hierarchy of equations for $n$-particle density matrices with proper boundary conditions can be derived from equation (2.10). Multiplying this equation by the operator $a_{l_{n}^{\prime}}^{\dagger} \cdots a_{l_{1}^{\prime}}^{\dagger} a_{l_{1}} \cdots a_{l_{n}}$ and then calculating the trace, we obtain [6]

$$
\begin{aligned}
\frac{\partial}{\partial t} f_{n}\left(l_{1} \ldots l_{n}, l_{1}^{\prime} \ldots l_{n}^{\prime} ; t\right)+\frac{\mathrm{i}}{\hbar}\left\langle\left[a_{l_{n}^{\prime}}^{\dagger} \ldots a_{l_{1}^{\prime}}^{\dagger} a_{l_{1}} \ldots a_{l_{n}}, H\right]\right\rangle^{t} \\
=-\varepsilon\left\{f_{n}\left(l_{1} \ldots l_{n}, l_{1}^{\prime} \ldots l_{n}^{\prime} ; t\right)-\bar{f}_{n}\left(l_{1} \ldots l_{n}, l_{1}^{\prime} \ldots l_{n}^{\prime} ; t\right)\right\}
\end{aligned}
$$

where

$$
\bar{f}_{n}\left(l_{1} \ldots l_{n}, l_{1}^{\prime} \ldots l_{n}^{\prime} ; t\right)=\operatorname{Tr}\left\{\varrho_{\text {rel }}(t) a_{l_{n}^{\prime}}^{\dagger} \cdots a_{l_{1}^{\prime}}^{\dagger} a_{l_{1}} \cdots a_{l_{n}}\right\}
$$

are the relevant density matrices, i.e. reduced density matrices in the relevant ensemble described by the statistical operator (2.14).

The source term on the right-hand side of equation (2.10) selects the retarded solution of the Liouville-von Neumann equation which coincides with the relevant

\footnotetext{
${ }^{1}$ For simplicity, we restrict our consideration to situations where the "anomalous" averages, like $\left\langle a_{l^{\prime}} a_{l}\right\rangle^{t}$, are zero. If this is not the case, the additional terms must be included into equation (2.14).
} 
distribution in a distant past $[2,3]$. Thus, the source terms in equations (2.17) define the corresponding boundary conditions for the reduced density matrices:

$$
f_{n}\left(l_{1} \ldots l_{n}, l_{1}^{\prime} \ldots l_{n}^{\prime} ; t\right)-\bar{f}_{n}\left(l_{1} \ldots l_{n}, l_{1}^{\prime} \ldots l_{n}^{\prime} ; t\right) \rightarrow 0, \quad t \rightarrow-\infty .
$$

These conditions imply, in fact, that the difference between the true nonequilibrium density matrices and their values in the relevant ensemble becomes negligible for times considerably greater than some correlation time.

It can be easily shown (see, e.g., [3]) that Bogoliubov's boundary condition of a complete weakening of initial correlations is recovered if we take $\varrho_{\text {rel }}(t)$ in the form (2.16). Keeping higher-order terms in the relevant statistical operator (2.14), more general boundary conditions can be formulated. The scheme with $f_{1}$ and $f_{2}$, taken as independent observables, was used, for example, to study two-particle correlations and the bound state formation in nonequilibrium Fermi systems [4,5].

Of special importance are long-lived many-particle correlations associated with the conservation laws. In the case of one-component systems, the locally conserved quantities are the densities of mass, momentum and energy. It should be emphasized that, in general, the average energy cannot be expressed in terms of the single-particle density matrix only, since the mean value of the interaction energy is given by the two-particle density matrix. Thus, for strongly correlated systems, the energy density must be included into the set of relevant variables. Assuming, for simplicity, that the system is spatially homogeneous and taking $\langle H\rangle^{t}$, instead of the total two-particle density matrix $f_{2}$, as a relevant observable together with the single-particle density matrix $f_{1}$, we have the relevant statistical operator [6]

$$
\varrho_{\text {rel }}(t)=\frac{1}{Z(t)} \exp \left\{-\beta(t)\left[H-\sum_{\boldsymbol{p}, \sigma} \mu_{\sigma}(\boldsymbol{p}, t) a_{\boldsymbol{p} \sigma}^{\dagger} a_{\boldsymbol{p} \sigma}\right]\right\} \text {. }
$$

Here we have chosen the momentum representation of single-particle states, $|l\rangle=$ $|\boldsymbol{p}, \sigma\rangle$, where $\boldsymbol{p}$ is the momentum variable and $\sigma$ is the spin index. The Lagrange multipliers $\beta(t)$ and $\mu_{\sigma}(\boldsymbol{p}, t)$ are determined from the self-consistency conditions

$$
\langle H\rangle^{t}=\operatorname{Tr}\left\{\varrho_{\mathrm{rel}}(t) H\right\}, \quad\left\langle a_{\boldsymbol{p} \sigma}^{\dagger} a_{\boldsymbol{p} \sigma}\right\rangle^{t}=\operatorname{Tr}\left\{\varrho_{\mathrm{rel}}(t) a_{\boldsymbol{p} \sigma}^{\dagger} a_{\boldsymbol{p} \sigma}\right\} .
$$

As the system goes to equilibrium, the parameters $\beta(t)$ and $\mu_{\sigma}(\boldsymbol{p}, t)$ tend to the equilibrium inverse temperature $T^{-1}$ and the chemical potential $\mu$, respectively. It is interesting to note that, taking the relevant statistical operator $(2.20)$ to define boundary conditions for the quantum BBGKY hierarchy, one obtains for the single-particle matrix a kinetic equation which is a quantum generalization of the well-known classical Enskog equation [6].

We have outlined a way toward resolving the problem of many-particle correlations in the Schrödinger picture of the quantum kinetic theory. To summarize briefly, long-lived many-particle correlations can be incorporated through boundary conditions for the reduced density matrices in the quantum hierarchy. These conditions are, in their turn, formulated in terms of the auxiliary relevant statistical 
operator which gives the thermodynamic description of the nonequilibrium state. The chief merit of this approach is that the kinetic equation for the single-particle density matrix can be combined with the evolution equations for thermodynamic quantities like the nonequilibrium temperature. Another important advantage of this scheme is that, extending the set of relevant variables, we can use Markovian kinetic equations, since all the long-lived correlations are included through the boundary conditions.

It should be noted, however, that the Schrödinger picture of the quantum kinetic theory has some shortcomings. The main defect of this picture lies in the fact that the reduced density matrices are single-time quantities. As a consequence, the quantum BBGKY hierarchy is unsuitable for describing the spectral properties of many-time (dynamical) correlations. Another weak point of the Schrödinger picture is that up to now we have no satisfactory technique for partial summations over infinite sets of terms in the quantum BBGKY hierarchy, which is necessary in the cases where a simple truncation procedure does not work. As we shall see below, some way out can be found if we combine the Schrödinger and the Heisenberg pictures of the quantum kinetic theory.

\section{Boundary conditions in the Heisenberg picture of the quan- tum kinetic theory}

Let us turn back to equations (1.1) which show that the time dependence of nonequilibrium averages can be analyzed by shifting the evolution operators from the statistical distribution to dynamical variables. We now want to formulate boundary conditions for the averaged products of Heisenberg operators (1.3) as $t_{0} \rightarrow-\infty$. For the purpose of the following consideration, it is convenient to introduce $n$-time products, i.e. the correlation functions

$$
\left\langle\hat{A}_{1 \mathrm{H}}\left(t_{1}\right) \cdots \hat{A}_{n \mathrm{H}}\left(t_{n}\right)\right\rangle^{t_{0}}=\operatorname{Tr}\left\{\varrho\left(t_{0}\right) \hat{A}_{1 \mathrm{H}}\left(t_{1}\right) \cdots \hat{A}_{n \mathrm{H}}\left(t_{n}\right)\right\}
$$

Note that the initial time $t_{0}$ appears not only in the statistical distribution, but also in all the evolution operators [see equation (1.3)]. This leads to some difficulties in taking the limit $t_{0} \rightarrow-\infty$ directly. Our first step is, therefore, to rewrite the correlation function in a more convenient form. Using the group property of the evolution operators, $U\left(t_{1}, t_{2}\right) U\left(t_{2}, t_{3}\right)=U\left(t_{1}, t_{3}\right)$, and the cyclic invariance of the trace, we obtain

$$
\begin{aligned}
& \left\langle\hat{A}_{1 \mathrm{H}}\left(t_{1}\right) \cdots \hat{A}_{n \mathrm{H}}\left(t_{n}\right)\right\rangle^{t_{0}}=\operatorname{Tr}\left\{U\left(\tau, t_{0}\right) \varrho\left(t_{0}\right) U\left(t_{0}, \tau\right)\right. \\
& \left.\quad \times U\left(\tau, t_{1}\right) \hat{A}_{1} U\left(t_{1}, t_{2}\right) \cdots U\left(t_{n-1}, t_{n}\right) \hat{A}_{n} U\left(t_{n}, \tau\right)\right\},
\end{aligned}
$$

where $\tau$ is an arbitrary instant of time. Now the choice of the initial time is related only to the evolution of the statistical operator. 
It is natural to consider $\varrho\left(t_{0}\right)$ in equation (3.2) as a retarded solution of the Liouville-von Neumann equation with the boundary condition for $t_{0} \rightarrow-\infty$ determined by some relevant distribution of the form (2.14). In other words, we will regard $\varrho\left(t_{0}\right)$ as a solution of equation (2.10). Note that the statistical operator, when defined in this way, satisfies the ergodic condition [7]

$$
U\left(t, t_{0}\right)\left\{\varrho\left(t_{0}\right)-\varrho_{\text {rel }}\left(t_{0}\right)\right\} U\left(t_{0}, t\right) \rightarrow 0, \quad t-t_{0} \rightarrow \infty .
$$

Then, from equations (3.2) and (3.3) we obtain the boundary condition for the averaged products of Heisenberg operators [8]:

$$
\left\langle\hat{A}_{1 \mathrm{H}}\left(t_{1}\right) \cdots \hat{A}_{n \mathrm{H}}\left(t_{n}\right)\right\rangle^{t_{0}} \rightarrow\left\langle\hat{A}_{1 H}\left(t_{1}\right) \cdots \hat{A}_{n H}\left(t_{n}\right)\right\rangle_{\mathrm{rel}}^{t_{0}}, \quad t_{0} \rightarrow-\infty .
$$

Relation (3.3) can be represented in another form using Abel's theorem, i.e. replacing the $t$-limit by the so-called $\varepsilon$-limit $[3,7]$. This gives

$$
\begin{aligned}
& \lim _{t_{0} \rightarrow-\infty} U\left(\tau, t_{0}\right) \varrho\left(t_{0}\right) U\left(t_{0}, \tau\right) \\
& \quad=\lim _{\varepsilon \rightarrow+0} \varepsilon \int_{-\infty}^{\tau} \mathrm{d} t_{0} \mathrm{e}^{-\varepsilon\left(\tau-t_{0}\right)} U\left(\tau, t_{0}\right) \varrho_{\text {rel }}\left(t_{0}\right) U\left(t_{0}, \tau\right) .
\end{aligned}
$$

Thus, instead of equation (3.4), we may use the boundary condition

$$
\begin{aligned}
\lim _{t_{0} \rightarrow-\infty}\left\langle\hat{A}_{1 \mathrm{H}}\left(t_{1}\right)\right. & \left.\cdots \hat{A}_{n \mathrm{H}}\left(t_{n}\right)\right\rangle^{t_{0}} \\
& =\lim _{\varepsilon \rightarrow+0} \varepsilon \int_{-\infty}^{\tau} \mathrm{d} t_{0} \mathrm{e}^{-\varepsilon\left(\tau-t_{0}\right)}\left\langle\hat{A}_{1 \mathrm{H}}\left(t_{1}\right) \cdots \hat{A}_{n \mathrm{H}}\left(t_{n}\right)\right\rangle_{\mathrm{rel}}^{t_{0}} .
\end{aligned}
$$

We want to point out one consequence of this condition. As already discussed, in the kinetic theory the quantities of interest are the reduced density matrices which are single-time averages of the creation and annihilation operators. Putting $\tau=t_{1}=t_{2}=\ldots=t_{n}=t$ in equation (3.6), we find the single-time averages in terms of Heisenberg operators averaged over the relevant ensemble [8]:

$$
\left\langle\hat{A}_{1} \cdots \hat{A}_{n}\right\rangle^{t}=\left.\lim _{\varepsilon \rightarrow+0} \varepsilon \int_{-\infty}^{t} \mathrm{~d} t_{0} \mathrm{e}^{-\varepsilon\left(t-t_{0}\right)}\left\langle\hat{A}_{1 \mathrm{H}}\left(t_{1}\right) \cdots \hat{A}_{n \mathrm{H}}\left(t_{n}\right)\right\rangle_{\mathrm{rel}}^{t_{0}}\right|_{t_{1}=\ldots=t_{n}=t} .
$$

This equation has two important aspects. First, it relates the nonequilibrium averages to time correlation functions in the relevant ensemble where long-lived correlations are incorporated. Second, we shall see below that, for Fermi and Bose systems, the time correlation functions appearing on the right-hand side of equation (3.7) can be calculated from the nonequilibrium Green functions.

\section{Boundary conditions in the real-time Green function formalism}

The real-time Green function technique for constructing kinetic equations was proposed by Kadanoff and Baym [9] and then developed by many authors. The 
extensive literature on this subject is reviewed, e.g., in [10,11]. We want to discuss some aspects of the Green function formalism related to the inclusion of long-lived correlations into the quantum kinetic theory.

In this section we assume that the system of identical fermions or bosons is described by the second quantized field operators $\psi(r)$ and $\psi^{\dagger}(r)$, where the argument $r$ involves all single-particle quantum numbers, say, the position vector $\boldsymbol{r}$ and the spin index $\sigma$. We also use a short notation $(k)=\left(r_{k}, t_{k}\right)$ and $\left(k^{\prime}\right)=\left(r_{k}^{\prime}, t_{k}^{\prime}\right)$ for the arguments of Heisenberg operators.

In the standard real-time Green function formalism, the basic quantity is the path-ordered single-particle Green function

$$
G\left(1,1^{\prime} ; t_{0}\right)=(\mathrm{i} \hbar)^{-1}\left\langle T_{\mathrm{C}}\left[\psi_{\mathrm{H}}(1) \psi_{\mathrm{H}}^{\dagger}\left(1^{\prime}\right)\right]\right\rangle^{t_{0}},
$$

where $T_{\mathrm{C}}$ is the path-ordering operator on the directed Keldysh-Schwinger contour $C$ shown in figure 1 (as usual, the ordering procedure includes the sign convention for the permutation of Fermi operators). Many-particle Green functions are intro-

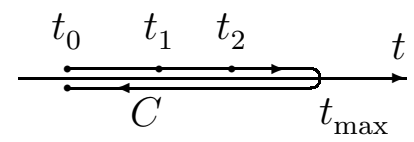

Figure 1. The directed Keldysh-Schwinger contour $C$ with the upper (chronological) and lower (anti-chronological) branches, $C^{+}$and $C^{-}$. The contour runs above the largest argument of the Green function. For instance, one may take the limit $t_{m} \rightarrow \infty$.

duced in a similar way. For instance, the $n$-particle Green function on the contour $C$ is defined as

$$
G\left(1 \ldots n, 1^{\prime} \ldots n^{\prime} ; t_{0}\right)=(\mathrm{i} \hbar)^{-n}\left\langle T_{\mathrm{C}}\left[\psi_{\mathrm{H}}(1) \cdots \psi_{\mathrm{H}}(n) \psi_{\mathrm{H}}^{\dagger}\left(n^{\prime}\right) \cdots \psi_{H}^{\dagger}\left(1^{\prime}\right)\right]\right\rangle^{t_{0}} .
$$

In many cases it is convenient to consider the single-particle Green function as a matrix (the fixed argument $t_{0}$ is omitted for brevity):

$$
G\left(1,1^{\prime}\right)=\left(\begin{array}{ll}
G^{++}\left(1,1^{\prime}\right) & G^{+-}\left(1,1^{\prime}\right) \\
G^{-+}\left(1,1^{\prime}\right) & G^{--}\left(1,1^{\prime}\right)
\end{array}\right)=\left(\begin{array}{cc}
g^{\mathrm{c}}\left(1,1^{\prime}\right) & g^{<}\left(1,1^{\prime}\right) \\
g^{>}\left(1,1^{\prime}\right) & g^{\mathrm{a}}\left(1,1^{\prime}\right)
\end{array}\right)
$$

The causal, $g^{\mathrm{c}}$, anti-causal, $g^{\mathrm{a}}$, Green functions and the correlation functions $g^{>,<}$ are given by

$$
\begin{aligned}
& g^{\mathrm{c}, \mathrm{a}}\left(1,1^{\prime} ; t_{0}\right)=(\mathrm{i} \hbar)^{-1}\left\langle T^{\mathrm{c}, \mathrm{a}}\left[\psi_{\mathrm{H}}(1) \psi_{\mathrm{H}}^{\dagger}\left(1^{\prime}\right)\right]\right\rangle^{t_{0}}, \\
& g^{>}\left(1,1^{\prime} ; t_{0}\right)=(\mathrm{i} \hbar)^{-1}\left\langle\psi_{\mathrm{H}}(1) \psi_{\mathrm{H}}^{\dagger}\left(1^{\prime}\right)\right\rangle^{t_{0}}, \\
& g^{<}\left(1,1^{\prime} ; t_{0}\right)=\mp(\mathrm{i} \hbar)^{-1}\left\langle\psi_{\mathrm{H}}^{\dagger}\left(1^{\prime}\right) \psi_{\mathrm{H}}(1)\right\rangle^{t_{0}} .
\end{aligned}
$$

As before, the upper (lower) sign refers to fermions (bosons). The symbols $T^{\text {,a }}$ denote the usual chronological and anti-chronological time ordering with the sign convention for fermions. 
Clearly, the real-time Green functions depend on the initial statistical operator $\varrho\left(t_{0}\right)$. Unless the state of the system is specially prepared at time $t_{0}$, the nonphysical dependence of the Green functions on the initial state must be eliminated. It is natural to take the limit $t_{0} \rightarrow-\infty$, since a macroscopic system "forgets" the non-relevant details of its initial state after some microscopic correlation time $\tau_{\mathrm{c}}$ which is characteristic of a given system.

In the standard theory of the real-time Green functions, it is assumed that in the limit $t_{0} \rightarrow-\infty$ the system is in a non-correlated state, i.e. is described by the statistical distribution

$$
\varrho_{\text {rel }}\left(t_{0}\right)=\frac{1}{Z\left(t_{0}\right)} \exp \left\{-\int \mathrm{d} r_{1} \mathrm{~d} r_{1}^{\prime} s_{1}\left(r_{1}^{\prime}, r_{1} ; t_{0}\right) \psi^{\dagger}\left(r_{1}^{\prime}\right) \psi\left(r_{1}\right)\right\},
$$

which is nothing but the relevant distribution (2.16) written in terms of the field operators. Since the limiting distribution admits Wick's decomposition, the limiting $n$-particle density matrices are uncoupled to single-particle density matrices. This leads to some boundary conditions for many-particle Green functions (4.2). In particular, the two-particle Green function satisfies the boundary condition (see, e.g., [11])

$$
\begin{aligned}
& \lim _{t_{0} \rightarrow-\infty} G\left(12,1^{+} 2^{+} ; t_{0}\right) \mid \begin{array}{l}
t_{1}=t_{2}=t_{0} \\
t_{1}^{\prime}=t_{2}^{\prime}=t_{0}
\end{array} \\
& \quad=\lim _{t_{0} \rightarrow-\infty}\left[G\left(1,1^{+} ; t_{0}\right) G\left(2,2^{+} ; t_{0}\right) \mp G\left(1,2^{+} ; t_{0}\right) G\left(2,1^{+} ; t_{0}\right)\right],
\end{aligned}
$$

where the notation $(k)^{+}=\left(r_{k}, t_{k}^{+}\right)$indicates that time $t_{k}^{+}$is taken infinitesimally later on the the Keldysh-Schwinger contour, than $t_{k}$.

The boundary condition (4.8) is equivalent to the Bogoliubov condition of a complete weakening of initial correlations in a distant past and is, in fact, implied in the standard real-time Green function technique, although it is not always pointed out explicitly. Nevertheless, this condition is the primary source of difficulties in an attempt to go beyond the simple quasiparticle picture. As an illustration, let us consider the two-time average $\left\langle\hat{A}_{1 \mathrm{H}}\left(t_{1}\right) \hat{A}_{2 \mathrm{H}}\left(t_{2}\right)\right\rangle^{t_{0}}$ calculated over some statistical distribution $\varrho\left(t_{0}\right)$. We can separate variables describing "slow" and "fast" processes by means of the relations $t=\left(t_{1}+t_{2}\right) / 2$ and $\tau=t_{1}-t_{2}$, where the time argument $t$ corresponds to the slow evolution, while the argument $\tau$ may be thought of as describing fast processes caused by collisions between particles. It is clear that the average under consideration does not depend on the initial distribution $\varrho\left(t_{0}\right)$, only if the difference $t-t_{0}$ is much greater than the characteristic correlation time $\tau_{\mathrm{c}}$ for all the relevant correlations in the system. If we deal with long-lived correlations, the time $\tau_{\mathrm{c}}$ is large compared with the collision time $\tau_{\text {coll }}$. A system with bound states provides a typical example of such a situation. Even if bound states are absent, long-lived correlations may be caused by the dynamics of slow hydrodynamic modes or by slow relaxation processes in the system. Thus, in the case when the evolution of the system starts from a non-correlated initial state, one might expect significant memory effects in the equations for the Green functions. 
In practice, however, the memory effects are treated as small corrections ${ }^{2}$. That is why the standard real-time Green function technique works well only when the system can be described as a weakly interacting gas of quasiparticles.

One way to avoid the problem of memory effects in the theory of Green functions is the use of initial distributions $\varrho\left(t_{0}\right)$, including many-particle correlations. For a detailed discussion and the literature on this subject see, e.g., [14]. The weak point of this idea is that the initial correlations are assigned to some (usually nonphysical) state at a fixed time $t_{0}$, whereas the correlated state varies with time and its evolution is governed by macroscopic transport equations.

In order to take the evolution of the correlated state into account, we shall turn to our general relation (3.6) and formulate new boundary conditions for the realtime Green functions. Since the time $\tau$ in equation (3.6) may be chosen without regard to the order of the Heisenberg operators, the new boundary condition for the Green function (4.2) is

$$
\begin{aligned}
\lim _{t_{0} \rightarrow-\infty} G & \left(1 \ldots n, 1^{\prime} \ldots n^{\prime} ; t_{0}\right) \\
& =\lim _{\varepsilon \rightarrow+0} \varepsilon \int_{-\infty}^{\tau} \mathrm{d} t_{0} \mathrm{e}^{-\varepsilon\left(\tau-t_{0}\right)} G_{\mathrm{rel}}\left(1 \ldots n, 1^{\prime} \ldots n^{\prime} ; t_{0}\right),
\end{aligned}
$$

where the notation $G_{\text {rel }}$ denotes the Green function in the relevant ensemble at time $t_{0}$. This is a remarkable relation, because $G_{\text {rel }}$ depends on $t_{0}$ through macroscopic quantities describing the evolution of many-particle correlations. In the special case when the relevant distribution $\varrho_{\text {rel }}\left(t_{0}\right)$ corresponds to a non-correlated state [see equation (4.7], it is easy to verify that the boundary condition (4.8) follows from equation (4.9). Thus, one may consider equation (4.9) as a generalized condition of the weakening of initial correlations written in terms of real-time Green functions.

We will draw attention to one important consequence of equation (4.9). As it is evident from equation (4.6), the correlation function $g^{<}\left(1,1^{\prime} ; t_{0}\right)$ is of special interest in the kinetic theory; its value for $t_{1}=t_{1}^{\prime}$ gives a single-particle density matrix in the $r$-representation. In order to eliminate the dependence of $g^{<}$on the initial state, we have to take the limit $t_{0} \rightarrow-\infty$. Since this correlation function is a component of the matrix Green function (4.1), we may use the boundary condition (4.9). Thus, we get

$$
\begin{aligned}
f_{1}\left(r_{1}, r_{1}^{\prime} ; t_{1}\right) & =\left.\mp \mathrm{i} \hbar \lim _{t_{0} \rightarrow-\infty} g^{<}\left(1,1^{\prime} ; t_{0}\right)\right|_{t_{1}^{\prime}=t_{1}} \\
& =\left.\mp \mathrm{i} \hbar \lim _{\varepsilon \rightarrow+0} \varepsilon \int_{-\infty}^{t_{1}} \mathrm{~d} t_{0} \mathrm{e}^{-\varepsilon\left(t_{1}-t_{0}\right)} g_{\text {rel }}^{<}\left(1,1^{\prime} ; t_{0}\right)\right|_{t_{1}^{\prime}=t_{1}} .
\end{aligned}
$$

This relation may be applied in different ways. First, differentiating it with respect to $t_{1}$, we recover the quantum hierarchy for the single-time quantities, i.e. for the reduced density matrices. Note that the corresponding boundary conditions will be reproduced automatically. Second, equation (4.10) relates the single-particle

\footnotetext{
${ }^{2}$ Note that even in this case the analysis is rather sophisticated (see, e.g., $\left.[12,13]\right)$.
} 
density matrix to the two-time quantity $g_{\text {rel }}^{<}\left(1,1^{\prime} ; t_{0}\right)$. This allows one to incorporate the spectral properties of microscopic dynamics and, through the relevant distribution, the many-particle correlations.

\section{The "mixed" Green functions}

The foregoing discussion illustrates that a natural way of constructing kinetic equations for strongly correlated quantum systems is to employ the Green functions $G_{\text {rel }}$ defined in the relevant ensemble ${ }^{3}$. As in the standard formalism, the starting point can be the Dyson equation for the single-particle Green function $G\left(1,1^{\prime} ; t_{0}\right)$ and a diagram representation of the corresponding mass operator.

In principle, the path-ordered Green functions in the relevant ensemble may be considered on the Keldysh-Schwinger contour (see figure 1), but this is not a suitable way. One reason is that, in general, the relevant distribution $\varrho_{\text {rel }}\left(t_{0}\right)$ contains the correlation terms and, therefore, the Wick theorem cannot be applied directly. Apart from technical difficulties, there is also a more serious reason why the initial statistical distribution (at the ends of the contour) should be taken in a non-correlated form. We recall that the mass operator (or the self-energy) in the Dyson equation can formally be expressed in terms of the two-particle Green function and the inverse single-particle Green function $G^{-1}\left(1,1^{\prime}\right)$ [11]. It should be emphasized, however, that the existence of the Dyson equation depends on whether the equation for the inverse Green function $G^{-1}\left(1,1^{\prime}\right)$ has a unique solution. A straightforward diagram analysis of the perturbation series for the single-particle Green function (see, e.g., [10]) shows that the Dyson equation exists if the initial distribution on the Keldysh-Schwinger contour admits Wick's decomposition. Thus, in this case one may conclude that $G^{-1}\left(1,1^{\prime}\right)$ exists and is unique. For other cases, the question of the existence of the inverse single-particle Green function is open. Based on the above considerations, it might be natural to work with the Green functions defined on the contour with a non-correlated state at the ends. We will briefly sketch how such Green functions can be introduced in the context of the kinetic theory (for a more detailed discussion see [8]).

We assume that the Hamiltonian of the system can be represented as the sum $H=H^{0}+H^{\prime}$, where $H^{0}$ is a free-particle Hamiltonian, and $H^{\prime}$ describes twoparticle interactions. Then, in terms of the field operators, the Hamiltonian is given by [cf. equation (2.7)]

$$
\begin{aligned}
H= & H^{0}+H^{\prime}=\frac{\hbar^{2}}{2 m} \int \mathrm{d} r_{1} \nabla_{1} \psi^{\dagger}\left(r_{1}\right) \cdot \nabla_{1} \psi\left(r_{1}\right) \\
& +\frac{1}{2} \int \mathrm{d} r_{1} \mathrm{~d} r_{2} \mathrm{~d} r_{1}^{\prime} \mathrm{d} r_{2}^{\prime} \Phi\left(r_{1}^{\prime} r_{2}^{\prime}, r_{1} r_{2}\right) \psi^{\dagger}\left(r_{2}^{\prime}\right) \psi^{\dagger}\left(r_{1}^{\prime}\right) \psi\left(r_{1}\right) \psi\left(r_{2}\right) .
\end{aligned}
$$

We also have to specify the form of the relevant distribution $\varrho_{\text {rel }}\left(t_{0}\right)$. It is convenient

\footnotetext{
${ }^{3}$ From this point on, all the Green functions will correspond to the relevant ensemble. For brevity, the index "rel" will be omitted.
} 
to write this distribution as

$$
\varrho_{\text {rel }}\left(t_{0}\right)=\exp \left\{-\hat{S}\left(t_{0}\right)\right\} / \operatorname{Tr} \exp \left\{-\hat{S}\left(t_{0}\right)\right\}
$$

where $\hat{S}\left(t_{0}\right)$ is usually called the entropy operator $[3,8]$. As it is seen from equation (2.14), the entropy operator is the sum $\hat{S}\left(t_{0}\right)=\hat{S}^{0}\left(t_{0}\right)+\hat{S}^{\prime}\left(t_{0}\right)$, where $\hat{S}^{0}$ is a singleparticle contribution, and $\hat{S}^{\prime}$ describes many-particle correlations. For simplicity, we will retain only the term corresponding to two-particle correlations. Thus, in the $r$-representation we have

$$
\begin{aligned}
& \hat{S}^{0}\left(t_{0}\right)=\int \mathrm{d} r_{1} \mathrm{~d} r_{1}^{\prime} s_{1}\left(r_{1}^{\prime}, r_{1} ; t_{0}\right) \psi^{\dagger}\left(r_{1}^{\prime}\right) \psi\left(r_{1}\right), \\
& \hat{S}^{\prime}\left(t_{0}\right)=\frac{1}{2} \int \mathrm{d} r_{1} \mathrm{~d} r_{2} \mathrm{~d} r_{1}^{\prime} \mathrm{d} r_{2}^{\prime} s_{2}\left(r_{1}^{\prime} r_{2}^{\prime}, r_{1} r_{2} ; t_{0}\right) \psi^{\dagger}\left(r_{2}^{\prime}\right) \psi^{\dagger}\left(r_{1}^{\prime}\right) \psi\left(r_{1}\right) \psi\left(r_{2}\right),
\end{aligned}
$$

where the Lagrange multipliers $s_{1}$ and $s_{2}$ are to be determined from the selfconsistency conditions

$$
\begin{aligned}
& f_{1}\left(r_{1}, r_{1}^{\prime} ; t_{0}\right)=\operatorname{Tr}\left\{\varrho_{\text {rel }}\left(t_{0}\right) \psi^{\dagger}\left(r_{1}^{\prime}\right) \psi\left(r_{1}\right)\right\} \\
& f_{2}\left(r_{1} r_{2}, r_{1}^{\prime} r_{2}^{\prime} ; t_{0}\right)=\operatorname{Tr}\left\{\varrho_{\text {rel }}\left(t_{0}\right) \psi^{\dagger}\left(r_{2}^{\prime}\right) \psi^{\dagger}\left(r_{1}^{\prime}\right) \psi\left(r_{1}\right) \psi\left(r_{2}\right)\right\}
\end{aligned}
$$

We now define the Heisenberg picture on the directed contour $\underline{C}$ in the $(t, x)$ plane (figure 2). Introducing the variable $(\xi)=(t, x)$ that specifies a point on the

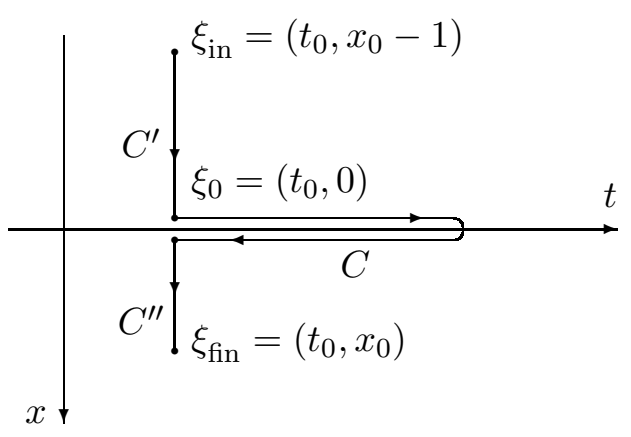

Figure 2. The directed contour $\underline{C}$ with the real-time evolution on the KeldyshSchwinger part $(C)$ and the "imaginary-time" evolution on the parts $C^{\prime}$ and $C^{\prime \prime}$. The parameter $x_{0}$ satisfies the inequality $0 \leqslant x_{0} \leqslant 1$.

contour $\underline{C}$, we write

$$
\hat{A}_{\mathrm{H}}(\xi)= \begin{cases}\mathrm{e}^{\mathrm{i}\left(t-t_{0}\right) H / \hbar} \hat{A} \mathrm{e}^{-\mathrm{i}\left(t-t_{0}\right) H / \hbar} & \text { on } C(\text { if } x=0), \\ \mathrm{e}^{x \hat{S}} \hat{A} \mathrm{e}^{-x \hat{S}} & \text { on } C^{\prime} \text { and } C^{\prime \prime}\left(\text { if } t=t_{0}\right),\end{cases}
$$

where the fixed parameter $t_{0}$ in the entropy operator is not written explicitly. It is clear that on the Keldysh-Schwinger part $(C)$ of the contour $\underline{C}$ we have the 
usual real-time Heisenberg picture and on the parts $C^{\prime}$ and $C^{\prime \prime}$ the above definition corresponds to the "imaginary-time" evolution governed by the entropy operator. To shorten the notation, from now on the underlined variables $(\underline{k})=\left(r_{k}, \xi_{k}\right)$ will be used to indicate that a function of such variables is defined on the directed contour $\underline{C}$. For the Keldysh-Schwinger part of the contour, the previous notation $(k)=\left(r_{k}, t_{k}\right)$ will be used.

With the definition (5.5) of the Heisenberg picture on the contour $\underline{C}$, we can introduce the corresponding path-ordered Green functions [8]. The single-particle Green function is defined as

$$
\underline{G}\left(\underline{1}, \underline{1}^{\prime} ; t_{0}\right)=(\mathrm{i} \hbar)^{-1}\left\langle T_{\underline{\mathrm{C}}}\left[\psi_{\mathrm{H}}(\underline{1}) \psi_{\mathrm{H}}^{\dagger}\left(\underline{1}^{\prime}\right)\right]\right\rangle_{\mathrm{rel}}^{t_{0}},
$$

where $T_{\underline{\mathrm{C}}}$ is the path-ordering operator on the contour $\underline{C}$. The path-ordered $n$ particle Green functions $\underline{G}\left(\underline{1}, \ldots, \underline{n}, \underline{1}^{\prime}, \ldots, \underline{n}^{\prime}\right)$ are introduced in perfect analogy to equation (4.2).

On the Keldysh-Schwinger part of the contour $\underline{C}$, the function (5.6) coincides with the real-time single-particle Green function [cf. equation (4.1)]. It should be recalled, however, that now the averaging is performed over the relevant distribution (5.2) but not over some unknown initial distribution $\varrho\left(t_{0}\right)$ as in equation (4.1). On the other hand, if the points $\xi_{1}$ and $\xi_{1}^{\prime}$ lie on the parts $C^{\prime}$ and $C^{\prime \prime}$ of the contour $\underline{C}$, then, up to a factor, the function (5.6) coincides with the so-called thermodynamic Green function which is a generalization of the well-known equilibrium Matsubara-Green function to the relevant ensembles [8]. To emphasize the above properties, the Green functions on the contour $\underline{C}$ will be referred to as the mixed Green functions.

To develop the perturbation theory with respect to many-particle terms $H^{\prime}$ and $\hat{S}^{\prime}$ in the Hamiltonian and the entropy operator, we define the interaction picture for the operators on the contour $\underline{C}$ :

$$
\hat{A}_{\mathrm{I}}(\xi)= \begin{cases}\mathrm{e}^{\mathrm{i}\left(t-t_{0}\right) H^{0} / \hbar} \hat{A} \mathrm{e}^{-\mathrm{i}\left(t-t_{0}\right) H^{0} / \hbar} & \text { on } C(\text { if } x=0), \\ \mathrm{e}^{x \hat{S}^{0}} \hat{A} \mathrm{e}^{-x \hat{S}^{0}} & \text { on } C^{\prime} \text { and } C^{\prime \prime}\left(\text { if } t=t_{0}\right) .\end{cases}
$$

Let us consider the product of operators $A_{1 \mathrm{H}}\left(\xi_{1}\right) A_{2 \mathrm{H}}\left(\xi_{2}\right) \cdots A_{k \mathrm{H}}\left(\xi_{k}\right)$ with the arguments arranged in a certain order on the contour $\underline{C}$. Recalling the definition (5.7) of the interaction picture, it is easy to verify the relation

$$
\hat{A}_{1 \mathrm{H}}\left(\xi_{1}\right) \cdots \hat{A}_{k \mathrm{H}}\left(\xi_{k}\right)=U_{\mathrm{I}}\left(\xi_{0}, \xi_{1}\right) \hat{A}_{1 \mathrm{I}}\left(\xi_{1}\right) U_{\mathrm{I}}\left(\xi_{1}, \xi_{2}\right) \cdots \hat{A}_{k \mathrm{I}}\left(\xi_{k}\right) U_{\mathrm{I}}\left(\xi_{k}, \xi_{0}\right),
$$

where $\xi_{0}=\left(t_{0}, 0\right)$ is a point at the junction of the parts $C, C^{\prime}$, and $C^{\prime \prime}$ of the contour (see figure 2). The interaction picture evolution operator $U_{I}\left(\xi, \xi^{\prime}\right)$ on $\underline{C}$ obeys the equations

$$
\frac{\partial U_{\mathrm{I}}\left(\xi, \xi^{\prime}\right)}{\partial \xi}=-\mathcal{H}_{\mathrm{I}}^{\prime}(\xi) U_{\mathrm{I}}\left(\xi, \xi^{\prime}\right), \quad \frac{\partial U_{\mathrm{I}}\left(\xi, \xi^{\prime}\right)}{\partial \xi^{\prime}}=U_{\mathrm{I}}\left(\xi, \xi^{\prime}\right) \mathcal{H}_{\mathrm{I}}^{\prime}\left(\xi^{\prime}\right)
$$


with the condition $U_{\mathrm{I}}(\xi, \xi)=1$. The effective "interaction Hamiltonian", $\mathcal{H}(\xi)$, is given by

$$
\mathcal{H}_{\mathrm{I}}^{\prime}(\xi)= \begin{cases}(\mathrm{i} / \hbar) H_{\mathrm{I}}^{\prime}(\xi)=(\mathrm{i} / \hbar) \mathrm{e}^{\mathrm{i}\left(t-t_{0}\right) H^{0} / \hbar} H^{\prime} \mathrm{e}^{-\mathrm{i}\left(t-t_{0}\right) H^{0} / \hbar} & \text { on } C, \\ \hat{S}_{\mathrm{I}}^{\prime}(\xi)=\mathrm{e}^{x \hat{S}^{0}} \hat{S}^{\prime} \mathrm{e}^{-x \hat{S}^{0}} & \text { on } C^{\prime}\left(C^{\prime \prime}\right) .\end{cases}
$$

A formal solution of equations (5.9) can be written in the form of the path-ordered exponent

$$
U_{\mathrm{I}}\left(\xi, \xi^{\prime}\right)=T_{\underline{\mathrm{C}}} \exp \left\{-\int_{\xi^{\prime}}^{\xi} \mathcal{H}_{\mathrm{I}}^{\prime}\left(\xi^{\prime \prime}\right) \mathrm{d} \xi^{\prime \prime}\right\}
$$

where the integral of the function $F(\xi)=F(t, x)$ along the contour $\underline{C}$ is defined as

$$
\begin{aligned}
\int_{\underline{C}} \mathrm{~d} \xi F(\xi)= & \left.\int_{C} \mathrm{~d} t F(t, 0)\right|_{\text {on } C} \\
& +\left.\int_{-1 / 2}^{0} \mathrm{~d} x F\left(t_{0}, x\right)\right|_{\text {on } C^{\prime}}+\left.\int_{0}^{1 / 2} \mathrm{~d} x F\left(t_{0}, x\right)\right|_{\text {on } C^{\prime \prime}}
\end{aligned}
$$

Equations (5.8) and (5.11) allow us to express the path-ordered products of the Heisenberg operators in terms of the path-ordered products in which the evolution is governed by the single-particle generators $H^{0}$ and $\hat{S}^{0}$. Note, however, that in the mixed Green function formalism we deal with the path-ordered products of the Heisenberg operators averaged over $\varrho_{\text {rel }}\left(t_{0}\right)$. Thus, to write such averages in the interaction picture, the relevant distribution must be expanded in the correlation part $\hat{S}^{\prime}$ of the entropy operator. Explicit calculations show [8] that

$$
\mathrm{e}^{-\hat{S}}=U_{\mathrm{I}}\left(\xi_{0}, \xi_{\text {in }}\right) \mathrm{e}^{-\hat{S}^{0}} U_{\mathrm{I}}\left(\xi_{\text {fin }}, \xi_{0}\right)
$$

where $\xi_{\text {in }}$ and $\xi_{\text {fin }}$ are the initial and the final points on the contour $\underline{C}$, respectively (see figure 2). Now relations (5.8), (5.11), and (5.13) can be combined to write the averaged products of the Heisenberg operators in the form:

$$
\begin{aligned}
& \left\langle T_{\underline{\mathrm{C}}}\left[\hat{A}_{1 \mathrm{H}}\left(\xi_{1}\right) \cdots \hat{A}_{k \mathrm{H}}\left(\xi_{k}\right)\right]\right\rangle_{\text {rel }}^{t_{0}} \\
& =\frac{\left\langle T_{\underline{\mathrm{C}}}\left[\exp \left\{-\int_{\underline{\mathrm{C}}} d \xi \mathcal{H}_{\mathrm{I}}^{\prime}(\xi)\right\} \hat{A}_{1 \mathrm{I}}\left(\xi_{1}\right) \cdots \hat{A}_{k \mathrm{I}}\left(\xi_{k}\right)\right]\right\rangle_{0}^{t_{0}}}{\left\langle T_{\underline{\mathrm{C}}} \exp \left\{-\int_{\underline{\mathrm{C}}} d \xi \mathcal{H}_{\mathrm{I}}^{\prime}(\xi)\right\}\right\rangle_{0}^{t_{0}}} .
\end{aligned}
$$

The averages on the right-hand side are calculated over the relevant statistical operator

$$
\varrho_{\text {rel }}^{0}\left(t_{0}\right)=\exp \left\{-\hat{S}^{0}\left(t_{0}\right)\right\} / \operatorname{Tr} \exp \left\{-\hat{S}^{0}\left(t_{0}\right)\right\}
$$


which admits Wick's decomposition. The denominator in equation (5.14) is the normalization constant, as it can be seen from equation (5.12).

The structure of equation (5.14) is typical of a diagram technique in the theory of Green functions. Each term in this expansion can be evaluated using the Wick theorem. In general, the corresponding Feynman rules for the diagram representation of the mixed Green functions depend on the particular form of the perturbation terms $H^{\prime}$ and $\widehat{S}^{\prime}$ in the Hamiltonian and the entropy operator. In our case [see equations (5.1) and (5.3)], these rules are, in fact, the same for all the parts of the contour $\underline{C}$.

Since the statistical operator (5.15) describes a non-correlated state and, consequently, the diagram summation can be applied to the mixed Green functions, we conclude that the single-particle Green function on the contour $\underline{C}$, equation (5.6), obeys the Dyson equation

$$
\underline{G}(\underline{1}, \underline{2})=\underline{G}_{0}(\underline{1}, \underline{2})+\int_{\underline{\mathrm{C}}} \mathrm{d} \underline{1}^{\prime} \mathrm{d} \underline{2}^{\prime} \underline{G}_{0}\left(\underline{1}, \underline{1}^{\prime}\right) \underline{\Sigma}\left(\underline{1}^{\prime}, \underline{2}^{\prime}\right) \underline{G}\left(\underline{2}^{\prime}, \underline{2}\right)
$$

where $\underline{G}_{0}(\underline{1}, \underline{2})$ is the zeroth-order mixed Green function ${ }^{4}$, and $\underline{\Sigma}\left(\underline{1}^{\prime}, \underline{2}^{\prime}\right)$ is the manycomponent mass operator on the contour $\underline{C}$. Some properties of the Dyson equation (5.16) are discussed in [8].

The existence of the Dyson equation is, of course, a very important property of the mixed Green function defined in the relevant ensemble, but this is not the end of the story. The next step is to extract equations for the correlation functions $g^{>,<}(1,2)$ (the so-called Kadanoff-Baym equations) and transform them into a kinetic equation for the single-particle density matrix or the Wigner function. An analogous procedure is developed in the standard real-time formalism $[10,11]$ but is a challenge in the case of strongly-correlated systems.

\section{Concluding remarks}

It is usual to consider the Green function formalism and the method of the nonequilibrium statistical operator as alternative approaches to the quantum kinetic theory. Our aim was to show that the combination of these methods appears to be useful in constructing kinetic equations for the systems with long-lived manyparticle correlations.

At the present stage, we cannot say, of course, that we have a perfect method for deriving kinetic equations, because little is known about the properties of the mixed Green functions and the corresponding Dyson equation in the case of strongly correlated systems. Nevertheless, the mixed Green function formalism seems to be a method of considerable promise, since many well-defined approximations in the standard method of real-time Green functions, such as the HartreeFock approximation and the $T$-matrix approximation for self-energy [11], can be extended to the mixed Green functions.

\footnotetext{
${ }^{4}$ It describes non-interacting particles in the non-correlated relevant ensemble.
} 
When working with the relevant ensembles, the essential point is the solution of self-consistency equations which play the role of nonequilibrium equations of state. As an example, we refer to equations (5.4). In principle, the self-consistency equations can be solved approximately, using the so-called thermodynamic Green functions which are the generalization of the Matsubara-Green functions to the nonequilibrium states described by the relevant distributions [8]. It is interesting to note that the thermodynamic Green functions appear in the mixed Green function formalism when all the arguments of the Green function are on the parts $C^{\prime}$ and $C^{\prime \prime}$ of the contour $\underline{C}$ (see figure 2). Since the "imaginary evolution" on the parts $C^{\prime}$ and $C^{\prime \prime}$ is governed by the entropy operator $\hat{S}$, which also enters into the relevant distribution, it is clear that the thermodynamic Green functions obey a closed Dyson equation in the sense that the components of the total mixed Green function with the arguments on the Keldysh-Schwinger part of the contour $\underline{C}$ do not enter into this equation. Thus, the nonequilibrium thermodynamic correlations in the relevant ensemble can be treated separately from the dynamical correlations. On the other hand, the thermodynamic correlations contribute to the Dyson equation for the real-time components of the mixed Green function through the cross functions with the arguments on different parts of $\underline{C}$.

Here we have presented a general formulation of boundary conditions for the quantum BBGKY hierarchy and the real-time Green functions with allowance made for long-lived many-particle correlations. In [6] the new boundary conditions for the quantum hierarchy were used in the derivation of a generalized Enskog-type kinetic equation for dense, strongly correlated systems. It would be interesting to derive an analogous equation in the mixed Green function formalism where the quasiparticle description can be incorporated in a more consistent way. Another interesting problem is the derivation of kinetic equations for systems with bound states and long-range correlations, such as non-ideal quantum plasmas. Finally, we note that the mixed Green function formalism can also be applied to stronglycorrelated superfluids and superconductors. In such cases the set of Green functions must include the so-called "anomalous" Green functions describing the condensate mode.

\section{References}

1. Bogoliubov N.N. Problems of Dynamic Theory in Statistical Physics. MoscowLeningrad, Gostekhizdat, 1946 (in Russian). Reprinted in: Studies in Statistical Mechanics, vol. 1 (ed. de Boer J. and Uhlenbeck G.E.). Amsterdam, North-Holland, 1962.

2. Zubarev D.N. Nonequilibrium Statistical Thermodynamics. New York, Consultant Bureau, 1974.

3. Zubarev D.N., Morozov V.G., Röpke G. Statistical Mechanics of Nonequilibrium Processes. Volume 1: Basic Concepts. Kinetic Theory. Berlin, Akademie Verlag, 1996.

4. Röpke G. Quantum-statistical approach to the electrical conductivity of dense, hightemperature plasma. // Phys. Rev. A, 1988, vol. 38, No. 6, p. 3001-3016.

5. Röpke G. Bound states and superfluidity in strongly coupled Fermion systems. // Ann. Physik., 1994, vol. 3, p. 145-162. 
6. Morozov V.G., Röpke G. Quantum kinetic equation for nonequilibrium dense systems. // Physica A, 1995, vol. 221, p. 511-538.

7. Zubarev D.N., Kalashnikov V.G. Equivalence of some methods in statistical mechanics of nonequilibrium processes. // Teor. Mat. Fiz., 1971, vol. 7, No. 3, p. 372-393.

8. Zubarev D.N., Morozov V.G., Röpke G. Statistical Mechanics of Nonequilibrium Processes. Volume 2: Relaxation and Hydrodynamic Processes. Berlin, Akademie Verlag, 1997.

9. Kadanoff L.P., Baym G. Quantum Statistical Mechanics. New York, Benjamin, 1962.

10. Danielewicz P. Quantum theory of nonequilibrium processes. I. // Ann. Phys. (NY), 1984, vol. 152, p. 239-304.

11. Bottermans W., Malfliet R. Quantum transport theory of nuclear matter. // Phys. Rep., 1990, vol. 198, No. 3, p. 115-194.

12. Morawetz K., Röpke G. Memory effects and virial corrections in nonequilibrium dense systems. // Phys. Rev. E, 1995, vol. 51, No. 5, p. 4246-4261.

13. Bornath Th., Kremp D., Kraeft W.D., Schlanges M. Kinetic equations for a nonideal quantum system. // Phys. Rev. E, 1996, vol. 54, No. 4, p. 3274-3284.

14. Schoeller H. A new transport equation for single-time Green's functions in an arbitrary quantum system. General formalism. // Ann. Phys. (NY), 1994, vol. 229, p. 273-319.

\title{
Багаточастинкові кореляції та граничні умови у квантовій кінетичній теорії
}

\author{
В.Г.Морозов ${ }^{1}$, Г.Репке ${ }^{2}$ \\ 1 Московський інститут радіоелектроніки та автоматики, \\ просп. Вернадського, 78, 117454 Москва, Росія \\ 2 Університет м. Ростока, фізичний факультет, \\ D-18051 Росток, Німеччина
}

\section{Отримано 17 листопада 1997 р.}

Проблема багаточастинкових кореляцій у різних підходах квантової кінетичної теорії розглядається на основі методу нерівноважного статистичного оператора Д.М.Зубарєва. Показано, що довгоживучі кореляції можуть бути враховані через граничні умови для зведених багаточастинкових матриць густини та нерівноважні функції Ґріна дійсного часу. В рамках методу функцій Ґріна граничні умови зручно формулюються в термінах "змішаних" функцій Ґріна, які визначаються прямим контуром з дійсним часом еволюції, що керується гамільтоніаном системи, та "уявним" часом еволюції, що керується оператором ентропії. Розклад за збуреннями змішаних функцій Ґріна отримано в термінах частини гамільтоніана, що відповідає за взаємодію, та кореляційної частини оператора ентропії.

Ключові слова: нерівноважний статистичний оператор, багаточастинкові кореляції, нерівноважні функції Ґріна, оператор ентропії

PACS: $05.30 . \mathrm{Ch}, 05.20 . \mathrm{dd}$ 